\title{
Benachteiligung wegen psychischer Krankheit?
}

Jürgen Fritze, Pulheim

psychoneuro 2005; 31 (4): 222-224

Unter anderem einige Berufsverbände und Fachgesellschaften ärztlicher und psychologischer Psychotherapeuten haben den Petitionsausschuss des Bundestages angerufen mit dem Ziel, eine Änderung des Versicherungsvertragsgesetzes (VVG) zu erreichen: Die rückblickende Reichweite von Gesundheitsfragen im Rahmen der vorvertraglichen Anzeigepflicht solle auf fünf Jahre begrenzt werden. Dies wird damit begründet, Antragsteller, die wegen psychischer Probleme oder Krankheiten in der Vergangenheit behandelt worden waren, fänden keinen privaten Versicherungsschutz insbesondere für Krankheit oder Berufsunfähigkeit. Die Betroffenen seien mangels Versicherungsschutz von Armut bedroht. Diesen Sorgen scheinen Missverständnisse zugrunde zu liegen.
$\mathrm{F}$ rühere psychische Krankheit schließt den Abschluss einer privaten Krankenversicherung (oder einer Berufsunfähigkeitsversicherung) grundsätzlich nicht aus. Nur in der Berufsunfähigkeitsversicherung wird im Rahmen der Risikoprüfung mit einer Ausschlussklausel für psychische Erkrankungen gearbeitet. Diese Klausel bedeutet aber nicht, dass Versicherungsschutz versagt würde; vielmehr besagt die Klausel, dass Berufsunfähigkeit wegen psychischer Krankheit vom Versicherungsschutz nicht erfasst wird. Damit soll gerade Versicherbarkeit ermöglicht werden. Berufsunfähigkeit aus anderen Krankheitsgründen fällt sehr wohl unter den Versicherungsschutz.

Versicherungsschutz für basale, die Existenz bedrohende Risiken zu gewährleisten, ist im Sozialstaat bisher Aufgabe der sozialen Sicherungssysteme, also nicht der priva- ten Vorsorge. Das Gesetz zur Reform der Renten wegen verminderter Erwerbsfähigkeit hat - mit Übergangsregelungen - zum 1. Januar 2001 für die gesetzliche Rentenversicherung das Konstrukt der Berufsunfähigkeitrente abgeschafft und ein einheitliches und abgestuftes System einer Erwerbsminderungsrente eingeführt. Wer auf dem allgemeinen Arbeitsmarkt auf Grund einer gesundheitlichen Einschränkung nur noch unter drei Stunden täglich arbeiten kann, erhält eine volle Erwerbsminderungsrente, wer noch drei Stunden bis unter sechs Stunden täglich arbeiten kann, erhält eine halbe Erwerbsminderungsrente. Tatsächlich liegt die Höhe dieser Rente unter derjenigen der früheren Berufsunfähigkeitsrente. Das Gesetz geht davon aus, dass Versicherte, die eine halbe Erwerbsminderungsrente beziehen, ihren Lebensunterhalt durch weiteres Ein- kommen sichern, z.B. durch Teilzeitarbeit - oder eben durch eine private Berufsunfähigkeitsversicherung. Jedenfalls bietet die Erwerbsminderungsrente einen Grundschutz, um vor Verelendung zu bewahren; die private Berufsunfähigkeitsversicherung (die privaten Versicherer haben den Berufsunfähigkeitsbegriff beibehalten) ergänzt den Schutz.

Private Krankenversicherung bietet einerseits als Vollversicherung Schutz substitutiv zur gesetzlichen Krankenversicherung, andererseits als Zusatzversicherung für spezielle Unterbringung (das sind Komfortleistungen) bzw. Chefarztbehandlung. Der Zugang zur substitutiven Vollversicherung wird - abgesehen von Beamten - durch die Versicherungspflichtgrenze eingeschränkt: Erst oberhalb eines Monatseinkommens von derzeit (2005) $3900 €$ besteht Wahlfreiheit zwischen gesetzlicher und privater Krankenversicherung. Interessent und privater Krankenversicherer handeln als gleichberechtigte Partner einen Versicherungsvertrag aus, der dem Privatrecht unterliegt. Beide Partner sind nicht zum Vertragsabschluss gezwungen. Kommt es nicht zu einem Vertragsabschluss, bleibt dem Antragsteller der Schutz der gesetzlichen Krankenversiche- 
rung erhalten. Die Zusatzversicherungen sind uneingeschränkt zugänglich.

\section{Risikoäquivalenz}

Private Krankenversicherungen sind im Unterschied zu gesetzlichen Krankenkassen Individualversicherungen, d.h. es gibt keine beitragsfreie Mitversicherung von Familienangehörigen. Die Beiträge der privaten Krankenversicherung werden nach dem Prinzip der individuellen Risikoäquivalenz kalkuliert. Die Risikoäquivalenz ist ein verfassungsrechtliches Gebot: Die Beiträge sollen in einem vernünftigen Verhältnis zu den Leistungen stehen. Risikoäquivalenz bedeutet, dass grundsätzlich die Summe der über die Vertragslaufzeit zu entrichtenden Beiträge des Versichertenkollektivs der Summe der für die verbleibende Lebenserwartung zu erwartenden individuellen Krankheitskosten zu entsprechen hat.

Die zu erwartenden Krankheitskosten müssen also vor Vertragsabschluss vorausgeschätzt werden, um den Beitrag sachgerecht kalkulieren zu können. Da mit steigendem Alter die Krankheitskosten steigen, sind die Beiträge desto höher, je höher das Eintrittsalter ist. Um Versicherte vor untragbar hohen Beiträgen im Alter zu schützen, wird durch einen Alterungszuschlag auf den Beitrag eine Rückstellung gebildet, also quasi ein Sparguthaben, aus dem die höheren Krankheitskosten im Alter finanziert werden. Dank dieser Rückstellungen (und der daraus erzielten Zinsgewinne) steigen die Beiträge mit dem Alter also nicht im gleichen Maße wie die Krankheitskosten. Das ermöglicht, dass künftige Generationen nicht mit den Krankheitskosten der älteren Generationen belastet werden. In dieser Generationengerechtigkeit unterscheidet sich die private Krankenversicherung von der gesetzlichen, die - jedenfalls bisher - nach dem Umlageprinzip finanziert wird.

Versicherbar sind grundsätzlich nur unvorhersehbare, zufällige Risiken. Wenn zum Zeitpunkt des Eintritts eine Krankheit besteht, wird dieses Prinzip verletzt, indem unmittelbar medizinische Leistungen in Anspruch genommen werden. Die damit verbundenen Kosten müssen also als Zuschlag im Beitrag berücksichtigt werden. Alternativ können für eindeutig vollkommen ausheilende Krankheiten - Karenzzeiten vereinbart werden. Wenn der Antragsteller in der Vergangenheit Krankheiten erlitten hat, so können diese mit einem erhöhten Risiko von Folgekrankheiten oder Wiedererkrankungen (und entsprechenden Kosten) verbunden sein. Hierfür sind Risikozuschläge zu kalkulieren. Also werden dem Antragsteller Fragen zu aktuellen und früheren Krankheiten gestellt. Wird diese vorvertragliche Anzeigepflicht verletzt, kann der Versicherer vom Vertrag zurücktreten. Ansonsten bleibt der private Krankenversicherer - anders als in anderen Versicherungssparten - lebenslang an den Vertrag gebunden. Nur dem Versicherten steht das Recht zur Kündung oder Vertragsänderung zu.

Grundsätzlich müssen "gute“ und „schlechte“ Risiken in einem Versichertenkollektiv zufällig verteilt sein, also einer Normalverteilung entsprechen. Häufen sich in einem Kollektiv „schlechte“ Risiken, so steigen zwangsläufig die Ausgaben und damit die Beiträge. Mit steigenden Beiträgen wird der Versicherer unattraktiv, verliert also seine Wettbewerbsfähigkeit. Es kann sich also ein Teufelskreis entwickeln: Je weniger neue „gute“ Risken der Versicherer gewinnt, desto stärker werden die Beiträge des Versichertenkollektivs weiter steigen, desto unattraktiver wird das Unternehmen. Es liegt also in der Verantwortung des Versicherers für seine Versicherten, solcher Antiselektion vorzubeugen.

\section{- Risikoselektion}

Der Antiselektion leistet die gesetzlich vorgegebene Versicherungspflichtgrenze Vorschub, indem sie eine Selektion vorschreibt. Für einen freiwillig gesetzlich Versicherten ist es - abgesehen von der Höhe der Beiträge - desto attraktiver, sich privat zu versichern, je eher er damit rechnet krank zu werden. Risikozuschläge begrenzen indirekt die Antiselektion. Darüber hinaus muss ein privater Krankenversicherer Ver- träge ablehnen bei nicht abschätzbaren Risiken, für die also ein Risikozuschlag nicht kalkulierbar ist. Das ist Risikoselektion. Faktisch bedeutet auch ein Risikozuschlag, der für den Antragsteller nicht finanzierbar ist, einen Ausschluss. Da es für Beamte keine Versicherungspflichtgrenze gibt und nicht geben muss (sie sind beihilfeberechtigt), bedarf es hier keiner Risikoselektion. Entsprechend hat sich die private Krankenversicherung für Beamte geöffnet.

\section{Psychische Krankheiten sind häufig und gut behandelbar}

Psychische Krankheiten zählen zu den häufigsten Krankheiten überhaupt. Aus dem deutschen Gesundheitssurvey (1998) hat sich ergeben, dass $42,6 \%$ der Bevölkerung (48,9\% der Frauen, 36,8\% der Männer) irgendwann im Laufe ihres Lebens an mindestens einer psychischen Krankheit leiden, innerhalb eines Jahres 31,1\%, innerhalb eines Monats immerhin $19,8 \%$. Nur $36,4 \%$ der Betroffenen erhalten innerhalb eines Jahres eine auf diese Erkrankung zielende medizinische Intervention, sei es weil sie keine Hilfe in Anspruch nehmen oder weil die Erkrankung nicht erkannt wird. Die Behandlungsraten variieren zwischen den Diagnosen. Sie liegen bei Psychosen (i.w. Schizophrenien) mit $72 \%$ am höchsten, gefolgt von der Panikkrankheit mit 58\%. Sie ist mit 29\% am geringsten bei Suchtleiden (i.w. Alkoholkrankheit). In den letzten 20 Jahren hat sich der Anteil psychischer Krankheiten an den Krankheitstagen rund verdreifacht: 1980 noch 2,5\%, 2003 7,5\%. Mit zunehmendem Trend tragen psychische Krankheiten inzwischen mit rund $30 \%$ zur vorzeitigen Berentung wegen Erwerbsunfähigkeit bei.

Die relativ geringen Behandlungsraten erschrecken auch insofern, als erhebliche Therapieerfolge zu erzielen sind. Z.B. lassen sich mit Antidepressiva bei rund 50\% der Kranken innerhalb einer Behandlungsdauer von 8 Wochen Vollremissionen erreichen. Allerdings neigen psychische Krankheiten zu späteren Rezidiven, d.h. Wiedererkranken mit erneuter Behandlungsbe- 
dürftigkeit. Diese Rezidivraten variieren abhängig von der Art der Erkrankung. Sie variieren auch bei „derselben“ Krankheit interindividuell erheblich. Psychotherapie ist nach Kriterien der Evidenz-basierten Medizin wirksam. Pharmakotherapie und Psychotherapie mindern signifikant die Rezidivrisiken mit unterschiedlichem Gewicht abhängig von der Indikation.

\section{Konsequenzen für den pri-} vaten Versicherungsschutz

Da also psychische Krankheiten derart häufig und gut behandelbar sind, stellt sich die Frage, ob es wirklich sinnvoll ist, in der Berufsunfähigkeitsversicherung diese Krankheitsart als Ursache von Berufsunfähigkeit vom Versicherungsschutz auszuschließen, denn damit bleibt ein wesentliches Kundeninteresse unbefriedigt. Allerdings kann man auch umgekehrt argumentieren, sie seien gerade wegen ihrer Häufigkeit auszuschließen. Je wahrscheinlicher ein Risiko ist, desto schwerer ist es versicherbar und desto höher werden die Beiträge.

Andererseits ist aber fraglich, ob ein Ausschluss anhand des Kriteriums früherer Behandlung angesichts der immer noch geringen Behandlungsrate überhaupt im Sinne der Versicherungsprinzipien zielführend sein kann. Darüber hinaus fragt sich, ob die inzwischen umfangreichen epidemiologischen Kenntnisse nicht doch die Kalkulation von Risikozuschlägen ermöglichen können. Es handelt sich um ein versicherungsmathematisches Problem. Hier kann man nur hoffen, dass einzelne Versicherer den Wettbewerbsvorteil entdecken, Risikozuschläge zu kalkulieren statt Berufsunfähigkeit wegen psychischer Krankheit vom Schutz auszuschließen.

In der privaten Krankenversicherung gibt es keinen Ausschluss wegen psychischer Krankheit, wenn auch im Einzelfall ein Versicherer das Risiko scheuen mag oder darauf verzichtet, den Antragsteller mit einem sachgerecht kalkulierten - hohen - Risikozuschlag zu konfrontieren. Wird ein Antrag wegen einer früheren psychischen Krankheit vom privaten Krankenversicherer abgelehnt, so mag das zwar diskriminierend wirken (ein gelegentlich zu hörender Vorwurf), ohne aber die Kriterien einer Diskriminierung zu erfüllen: Es handelt sich um Einzelfallentscheidungen, und diese gelten nicht spezifisch psychischen Krankheiten, sondern grundsätzlich jedweden Vorerkrankungen, bei denen die Kalkulation eines Risikozuschlages schwierig oder unmöglich erscheint. Das würde transparenter, wenn der Versicherer auf die Ablehnung zugunsten des Angebotes eines Risikozuschlages verzichten würde.

Eine gängige Kritik stößt sich daran, dass psychotherapeutische Behandlungen in Kindheit oder Jugend Dekaden später, in denen keine Behandlung in Anspruch genommen wurde, als Kriterium für den Ausschluss von einer privaten Krankenversicherung herhalten soll. Tatsächlich aber zielen derzeit die vorvertraglichen Gesundheitsfragen der meisten Versicherer bezüglich psychischer Krankheiten auf die zurückliegenden fünf bis 10 Jahre, was bereits der Forderung gegenüber dem Petitionsausschuss weitgehend entspricht. Allerdings fragt sich auch hier, ob ein Ausschluss anhand des Kriteriums früherer Behandlung angesichts der immer noch geringen Behandlungsrate zielführend sein kann. Schließlich ist von allen Beteiligten zu würdigen, dass gerade in der Kindheit und Jugend die Übergänge zwischen psychischen Krankheiten und Entwicklungsproblemen fließend sind, allerdings mit einem Trend zur „Medizinalisierung“. Im Einzelfall hängt es vom Zufall ab, ob ein „Problem“ zur Erziehungsberatung oder zum Arzt führt. Bei der Kalkulation der Risikozuschläge und der Entscheidung über einen Vertragsabschluss liegt es am Versicherer, zwischen alterstypischen Erziehungsproblemen einerseits und psychischen Krankheiten im engeren Sinne andererseits zu differenzieren.

\section{- Fazit}

Nichtversicherbarkeit in einer privaten Versicherung hinterlässt den Antragsteller nicht in einer existenziellen Notlage. Risikozuschläge auf den Beitrag sind unverzichtbar, um dem allgemeinen Versicherungsprinzip der Äquivalenz zu genügen.

Die Möglichkeit zur Ablehnung eines Antrages auf eine private Krankenversicherung ist bei unkalkulierbaren Risiken ein unverzichtbares Steuerungsinstrument, solange es eine gesetzliche Versicherungspflichtgrenze gibt; anders ist der daraus resultierenden Antiselektion nicht zu begegnen. Die inzwischen umfangreichen und validen epidemiologischen Kenntnisse über Häufigkeit, Behandlungsraten und Verlauf psychischer Krankheiten sollten heute erlauben, risikogerechte $\mathrm{Zu}$ schläge zu kalkulieren, so dass Ausschlüsse vermieden werden.

Allerdings können die versicherungsmathematisch notwendigen Risikozuschläge im Einzelfall so hoch sein, dass sie den Antragsteller überfordern, was im Endeffekt einer Nichtversicherbarkeit gleichkommt. Eine - wie gegenüber dem Petitionsausschuss gefordert - Begrenzung der Reichweite der vorvertraglich zu beantwortenden Gesundheitsfragen auf z.B. fünf Jahre hilft nicht weiter. Sie kann sogar kontraproduktiv sein, da sie die Kalkulation des Risikozuschlages erschwert, was zu unsachgerecht hohen Risikozuschlägen führen kann.

Entsprechend hat auch das Bundesjustizministerium in seiner Stellungnahme zur Petition keinen Änderungsbedarf für das VVG gesehen.

Korrespondenzadresse:

Prof. Dr. med. Jürgen Fritze Asternweg 65

50259 Pulheim 